\title{
A Hardware-in-the-Loop Test Rig for Development of Electric Vehicle Battery Identification and State Estimation Algorithms
}

\author{
Abbas Fotouhi ${ }^{1}$, Karsten Propp ${ }^{1}$, Lilantha Samaranayake ${ }^{2}$, Daniel J. Auger ${ }^{1}$, Stefano Longo ${ }^{1}$ \\ 1. Advanced Vehicle Engineering Centre, Cranfield University, Bedfordshire, MK43 OAL, UK \\ 2. Department of Electrical and Electronic Engineering, Faculty of Engineering, University of \\ Peradeniya, Sri Lanka
}

\begin{abstract}
This paper describes a hardware-in-the-loop (HIL) test rig for the test and development of electric vehicle battery parameterization and state-estimation algorithms in the presence of realistic real-world duty cycles. The rig includes two electric machines, a battery pack, a realtime simulator, a thermal chamber and a PC for human-machine interface. Other parts of a vehicle powertrain system are modelled and used in the real-time simulator. A generic framework has been developed for real-time battery measurement, model identification and state estimation. Measurements are used to extract parameters of an equivalent circuit network model. Outputs of the identification unit are then used by an estimation unit trained to find the relationship between the battery parameters and state-of-charge. The results demonstrate that even with a high noise level in measured data, the proposed identification and estimation algorithms are able to work well in real-time.
\end{abstract}

Keywords: battery modelling, electric powertrain, hardware-in-the-loop test, state-of-charge estimation, identification.

\section{Nomenclature}

$\begin{array}{llcl}A_{f} & \text { vehicle frontal area } & \text { SOC } & \text { battery state-of-charge } \\ C_{d} & \text { drag coefficient } & S O C_{0} & \text { battery initial state-of-charge } \\ C_{r} & \text { rolling resistance coefficient } & T & \text { temperature } \\ C_{t} & \text { battery total capacity } & T_{m} & \text { electric motor torque } \\ D_{a} & \text { driver's acceleration command } & T_{m, \max } & \text { electric motor's maximum torque } \\ D_{b} & \text { driver's brake command } & V_{O C} & \text { battery open-circuit voltage }\end{array}$




\begin{tabular}{|c|c|c|c|}
\hline$F_{b}$ & brake force & $V_{t}$ & measured battery terminal voltage \\
\hline$F_{b, \max }$ & maximum brake force & $\hat{V}_{t}$ & estimated battery terminal voltage \\
\hline$F_{t}$ & tractive force & $y\left(t_{k}\right)$ & real output at time $\mathrm{k}$ \\
\hline$g$ & the gravity & $\hat{y}\left(t_{k} \mid t_{k-1} ; \theta\right)$ & $\begin{array}{l}\text { predicted value of the output at time } \mathrm{k} \\
\text { using the parameter vector } \theta\end{array}$ \\
\hline$G$ & gear ratio & $\varepsilon$ & prediction error \\
\hline$i(t)$ & battery current & $\gamma$ & battery coulombic efficiency \\
\hline$I_{i}$ & battery current vector & $\theta$ & battery model's parameters vector \\
\hline$M_{v}$ & vehicle mass & $\rho$ & air density \\
\hline$P_{i}$ & a parameter of the battery model & $\eta_{\text {gear }}$ & efficiency of gearbox \\
\hline$R_{\mathrm{int}}$ & battery internal (total) resistance & $\eta_{m}$ & efficiency of electric motor \\
\hline$R_{w}$ & wheel radius & & \\
\hline
\end{tabular}

\section{Introduction}

Development of electrical energy storage systems plays a significant role in the vehicle electrification process. Among the existing technologies in this area, batteries are the most widely used and still an area of constant development. In addition to the development of various battery chemistries, it is vital to develop proper battery management systems (BMS) to operate the battery in practical applications. In an electric vehicle (EV), it is important to understand state-ofcharge (SOC) or 'remaining capacity', which is vital for any kind of range prediction. EV range estimation, safe battery charge/discharge and optimal usage of batteries, all depend on efficient and accurate battery models. Different approaches have been used in the literature for battery modelling, reviewed by Fotouhi et al. (2016-b). Among all battery modelling approaches, equivalent circuit network (ECN) models are fast enough to be used in real-time applications. Because of the low computational effort and relatively good precision, ECN models have been the subject of studies in a wide range (Marc et al., 2008; He et al., 2011) specifically for automotive application (Antaloae et al., 2012; Chiasson and Vairamohan, 2005; Schweighofer and Brasseur, 2003).

BMS algorithms are designed for optimal and safe use of a battery by monitoring and control. The monitoring process consists of real-time measurements of variables such as current, voltage and temperature, which are used to calculate some 'unmeasurable' variables like battery state-of- 
charge (SOC) and state-of-health (SOH). The simplest method of battery SOC calculation, called 'coulomb counting', works based on integration of the battery current over time to calculate the amount of energy flowing through the battery. Because of the practical limitations of coulomb counting such as cumulative noise effect and the need for a known initial condition, more advanced battery SOC estimation techniques such as Kalman Filter-based estimators (He et al., 2013; Thrun et al., 2005; Welch et al., 1995; Plett et al., 2004), sliding mode observers (Edwards et al., 1998; Kim, 2010), and fast model identification techniques (Fotouhi et al., 2017-a) have been developed in the literature.

While the SOC estimation for lead-acid batteries can be relatively simple (Piller et al. 2001), direct measurement of physical properties of the battery is possible, due to the battery's tolerance for overcharge and overdischarge to some extent. However, this is not the case for other battery types. Li-ion batteries for example are sensitive to overcharge and overdischarge. So, the SOC must be known when dynamic currents are applied, as in electric vehicles, to guarantee the safety and an optimal lifetime (Lu et al. 2013). Less uncertainty in the SOC estimation allows battery operations closer to its limits without compromising safety or reliability. For these reasons many SOC estimation methods were developed for Li-ion batteries with accuracies up to $1 \%$ (Cuma et al. 2015). Here, the estimation effort, and therefore the precision, also depends on the use. While most simple consumer electronics use basic methods, the SOC estimation for electric vehicles is usually more complex since it has to be robust against a wide range of user conditions and highly dynamic current profiles.

Before applying a battery model or estimation algorithm in a real EV, a battery simulation environment is essential for testing the developed models and algorithms. This study describes a hardware-in-the-loop (HIL) test rig for the test and development of electric vehicle battery parameterization and state-estimation algorithms. HIL testing of BMS algorithms is a common technique in the literature to validate the software functionality under real working condition (Barreras et al., 2016; Wang, 2014).

In most conventional battery simulators, the duty cycle (or 'load profile') is obtained from simple models of the powertrain components. For example, simplified models of electric machines are used, and the detail of power electronics is often neglected or treated as a simple efficiency map with ideal instantaneous switching (Lukic and Emado, 2003; Gao et al., 2007). 
This study avoids these limitations as real, physical components are used within the simulation loop. A brushless DC (BLDC) machine driven by three-phase AC is used, together with its associated MOSFET-based power electronics. This type of machine is common in electric vehicles, and it would be hard to obtain a computational model that was sufficiently representative of its real-world transient behaviour.

There are power source/sink devices available in the market which can be also used to charge/discharge a battery pack based on a real driving scenario. The quality of the measurements might be also better than the sensors used in this study. However, the high noise level in measurements in the proposed rig is an advantage for BMS development since it would be more challenging. Here the goal was to simulate the EV battery's working condition as much as possible similar to real working conditions. The proposed test rig represents a scaled-down EV powertrain system with even less expensive sensors that are used in a typical EV. This allows the testing of the BMS algorithms in an environment that is more representative of real-world dutycycles and thereby reduces the risk of failure at a later implementation stage.

The BMS algorithms development in this study mainly contains two parts: (i) using an ECN model, a fast system identification technique is applied to real-time parameterization of a battery pack, and (ii) the battery parameters are used for real-time battery state estimation. For this purpose, a generic framework has been developed including battery measurement, model identification and state estimation units. In the measurement unit, battery load current and terminal voltage are recorded to be used by the identification unit in order to extract the parameters of an ECN battery model. Outputs of the identification unit are then used by an estimation unit to estimate the battery SOC. The important point is that all these functions are performed in real-time, so all the algorithms should be fast enough. The proposed algorithms in this study are tested in real-time using the test rig and the results are discussed in section 4 . Battery test rig is explained in detail in section 2 and the identification and estimation techniques are presented in section 3 .

\section{Battery HIL Test Rig}

\section{2-1 HIL structure}

The HIL test rig and its main components are shown in Figure 1. It includes two back-to-back connected electric machines, the respective power electronic controllers, a target battery pack (in 
this case, containing NiMH cells), a lead-acid battery pack to provide independent power sourcing and sinking, a dSPACE real-time simulator, a thermal chamber and a PC for humanmachine interface. The test rig contains two BDLC machines of 5kW each. One of these - shown to the left in Figure 1 - is used to represent EV traction motor and the second machine is used to apply dynamic loads ('load' machine) representing the torques experienced in a vehicle-level duty cycle. The 'traction' machine is connected to the battery pack under test. The load machine is connected to a bi-directional power source consisting of a separate DC supply built from readily available lead-acid batteries.

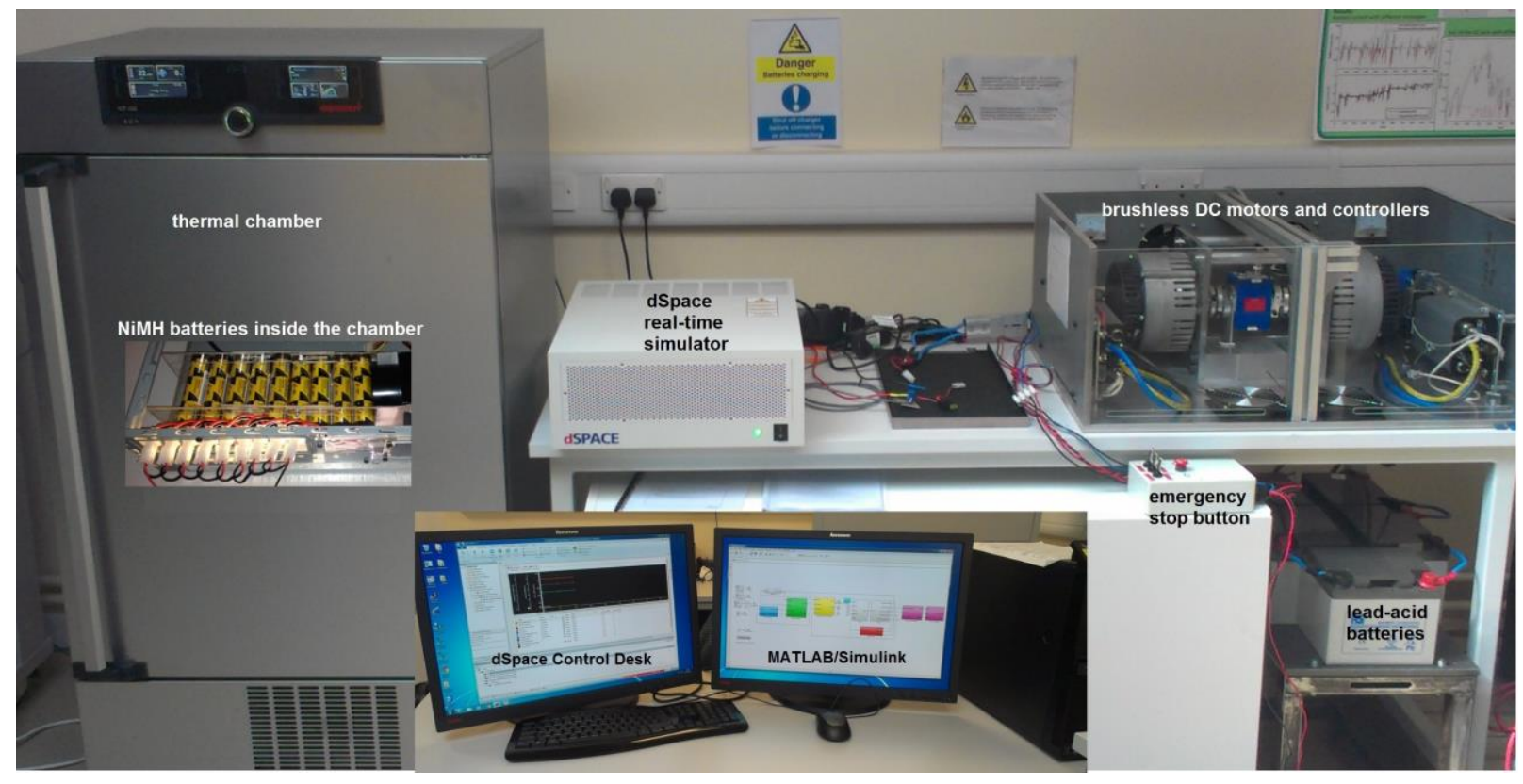

Figure 1: Battery HIL test rig and its components

\section{2-2 Electric Machines and motor controllers}

As mentioned above, $5 \mathrm{~kW}$ brushless DC (BLDC) machines are used in the battery test rig. A BLDC machine has a trapezoidal air gap flux distribution obtained through electronic commutation implemented through power electronics. In a three-phase ac machine of this type, a 'current controller' converts a 'torque reference' from an 'outer' speed controller to the respective current references for each of the three phase windings; to achieve this, the current controller outputs gate trigger signals to MOSFETs in the (hardware) motor controller block 
(Bose, 2001). The current controller employs nonlinear hysteresis type current comparators, which are the most rugged analog controllers. The rotor position required for the correct commutation of the windings is obtained from Hall-effect position sensors in the stator. The same rotor position signals are used to calculate the rotor speed. Since the machine has eight permanent magnet poles, three Hall-effect sensors are placed in the stator, 15 mechanical degrees apart. In the speed calculation algorithm, any change in the Hall-effect sensor output (corresponding to a rotor pole movement) is detected and the time between two consecutive such changes is calculated. The inverse of this time multiplied by the angle rotated (15 degrees) gives the rotational speed of the machine.

\section{2-3 Battery packs}

Two battery packs are used in the test rig: the 'main' battery pack under test (connected to the traction machine) and a 'secondary' lead-acid pack (connected to the load machine). The secondary pack provides the load and is not the subject of study: the role of the EV traction battery is supplied by the main battery pack. The secondary pack consists of five $12 \mathrm{~V}$ lead-acid batteries connected in series, giving a nominal $60 \mathrm{~V}$ which matches the motor controller's voltage range. In this study, for the main battery pack under test, a NiMH battery chemistry has been selected as a first attempt while keeping this option to replace it with any other battery type in the same scale. The NiMH battery pack was built with the general aim to simplify development of the test rig but still being sufficiently representative of the automotive context. NiMH batteries have advantages in experimental development due to their high safety in charge and discharge and tolerance to abuse (overcharge and overdischarge), their good volumetric energy, power and thermal properties, and their simple and inexpensive charging and control circuits (Fetcenko et al., 2007). As an initial configuration, radio-controlled car cell packs (with six cells each) were used with their original charging power supplies, since they charge the packs similarly to a defined point. Here nine modules in series (shown in Figure 2) are used to create a nominal voltage $(64.8 \mathrm{~V})$ within the window of the motor controller. The layout of six NiMH cells per module, connected in series, is particularly close to automotive applications since it is also used in hybrid cars (Kelly et al., 2002). Table 1 summarizes the battery pack configuration for Honda Insight and Toyota Prius from (Kelly et al., 2002), together the pack used here. 


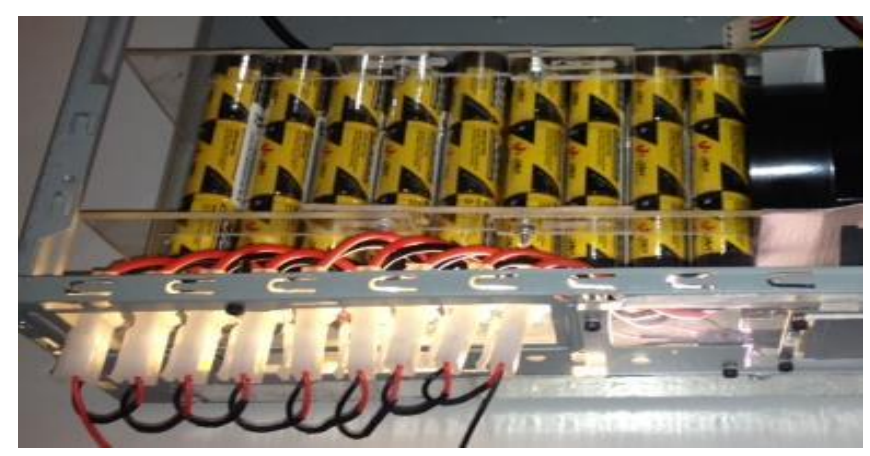

Figure 2: NiMH battery pack consisting of nine modules

Table 1: HIL battery pack configuration in comparison to two real EV battery packs

\begin{tabular}{rccc}
\hline & Honda Insight & Toyota Prius & Test Rig Pack \\
\hline Battery Type & NiMH & NiMH & NiMH \\
Nominal cell voltage & $1.2 \mathrm{~V}$ & $1.2 \mathrm{~V}$ & $1.2 \mathrm{~V}$ \\
Rated capacity & $6.5 \mathrm{Ah}$ & $6.5 \mathrm{Ah}$ & $5 \mathrm{Ah}$ \\
Cells per module & 6 & 6 & 6 \\
Number of modules & 20 & 38 & 9 \\
Total voltage & $144 \mathrm{~V}$ & $273.6 \mathrm{~V}$ & $64.8 \mathrm{~V}$ \\
Nominal energy storage & $936 \mathrm{Wh}$ & $1778 \mathrm{Wh}$ & $324 \mathrm{Wh}$ \\
\hline
\end{tabular}

\section{2-4 Simulation model}

The parts of the vehicle not represented by the test rig are implemented in software simulation model in MATLAB and Simulink. A typical EV model is developed which has been parameterized to represent the Nissan LEAF with specifications listed in Table 2 (Choi and Jahns, 2013; Hayes et al., 2011). The rig is used to simulate vehicle-level driving cycles such as the well-known urban dynamometer driving schedule (UDDS) (DieselNet, 2017), and because of the inclusion of physical components, it provides a good representative electrical load at battery level. As the components used in the test rig are not full size, appropriate scaling has been implemented to ensure that torques and speeds are appropriately matched to the components in use.

As illustrated in Figure 3, the first part of the proposed model is a driver model to follow the drive cycle. The driver model uses the drive cycle and velocity as inputs, and calculates an acceleration/deceleration command $\left(D_{a}\right.$ or $\left.D_{b}\right)$ as output. The driver model is a ProportionalIntegral (PI) controller tuned using Ziegler-Nichols method (Ziegler and Nichols, 1942) as 
discussed by Fotouhi et al. (2014). The output is scaled between 0 and 1 showing released and pushed pedal statuses.

The vehicle dynamics block in Figure 3, contains different parts as follows. The required tractive force $\left(F_{t}\right)$ is simply calculated based on electric motor's (EM) maximum torque and the driver's command as follows:

$$
\begin{gathered}
F_{t}=\eta_{m} \cdot \eta_{\text {gear }} \cdot \frac{G \cdot T_{\mathrm{m}}}{R_{w}} \\
T_{m}=D_{a} \cdot T_{m, \max }
\end{gathered}
$$

where $\eta_{m}$ and $\eta_{\text {gear }}$ are efficiencies of EM and gearbox respectively, $R_{w}$ is wheel radius, $G$ is gear ratio, $T_{m}$ is EM's required torque and $T_{m, \max }$ is EM's maximum torque calculated based on EM's torque-speed map. Having the tractive force value, vehicle's acceleration and speed are calculated regarding the opposing forces including air drag, rolling resistance, road gradient and brake forces. The coefficients' values are listed in Table 2. Brake force $\left(F_{b}\right)$ is calculated as follows:

$$
F_{b}=D_{b} \cdot F_{b, \max }
$$

where $F_{b, \max }$ is the maximum friction brake force.

The summation of the opposing forces is produced by the load machine (right EM) using a reference torque calculated in vehicle dynamics block as demonstrated in Figure 3. A torque sensor measures the real torque between the EMs and the measured signal (EM torque in Figure 3 ) is feed backed to the control block which is responsible to control the torque. On the other hand, the traction machine (left EM) should follow the speed. A speed sensor measures the EM's RPM and this signal is used by a speed controller to follow the reference RPM calculated in vehicle dynamics block. Both speed and torque controllers are Proportional-Integral (PI) type controllers tuned using Ziegler-Nichols method. The speed tracking results are presented and discussed in section 4. For the sake of simplicity and the limitations of capacity and maximum current of the NiMH battery pack, a small constant torque is considered. Otherwise, the battery pack would be subjected to high current and be depleted very quickly since there are not parallel circuits in the pack. In a full-scale battery pack test, the required current would be distributed 
between parallel circuits. Because the main goal of this study was to run and validate the identification and estimation algorithms in real-time, full-scale battery pack testing was not necessary so, the small pack described in section 2.3 was tested.

The controllers' outputs are acceleration/deceleration commands sent to the motor controllers in the rig. The motor controllers run the machines according to the commands and consequently the target battery pack is tested under real driving condition. The test continues by repeating the drive cycle until the battery is depleted. Battery depletion is recognized when battery terminal voltage drops below the cut-off voltage, i.e. $54 \mathrm{~V}$ in this case.

The last block is related to battery model identification and state estimation using the real-time measurements as demonstrated in Figure 3. This block has two inputs, current and voltage of the battery pack, and a number of outputs depending on the battery model's structure. Battery SOC is the main output of this block and is estimated by the proposed algorithms explained in more detail in section 3 .

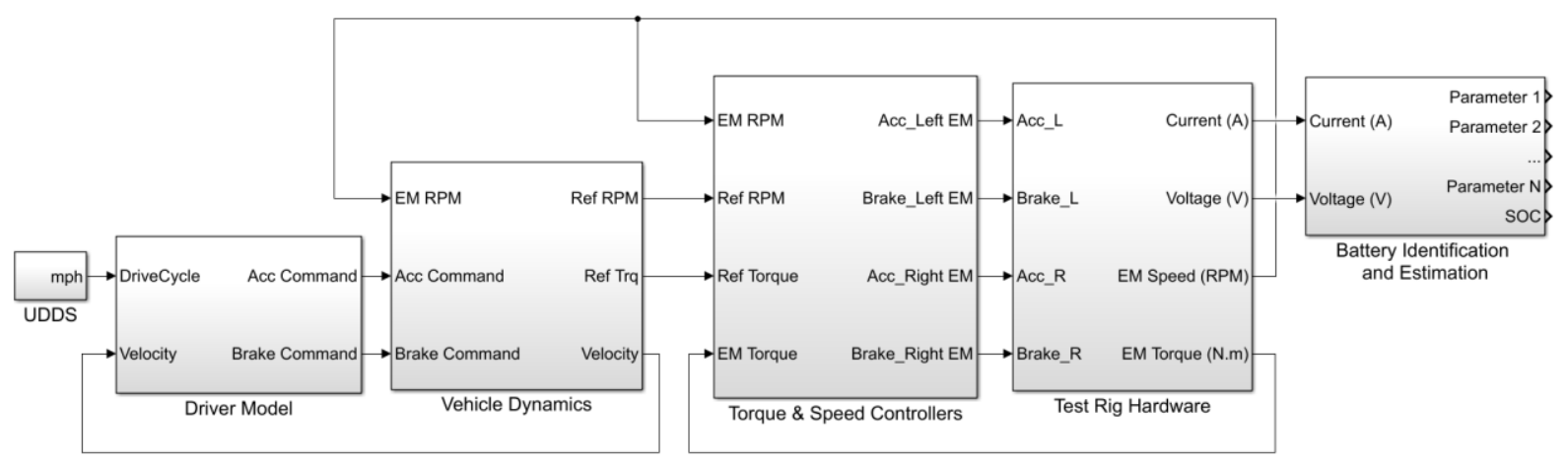

Figure 3: MATLAB-Simulink blocks used in test rig 
Table 2: Numerical values of EV model parameters

\begin{tabular}{|c|c|c|c|}
\hline Parameter & Description & Value & Unit \\
\hline$A_{f}$ & Vehicle frontal area & 2.27 & $\mathrm{~m}^{2}$ \\
\hline$C_{d}$ & Drag coefficient & 0.29 & - \\
\hline$C_{r}$ & $\begin{array}{l}\text { Rolling resistance } \\
\text { coefficient }\end{array}$ & 0.012 & - \\
\hline$F_{b, \max }$ & Maximum brake force & 3500 & $\mathrm{~N}$ \\
\hline$g$ & Gravity & 9.81 & $\mathrm{~m} / \mathrm{s}^{2}$ \\
\hline$G$ & Gear ratio & 7.94 & - \\
\hline$M_{v}$ & Vehicle mass & 1685 & $\mathrm{~kg}$ \\
\hline$R_{w}$ & Wheel's radius & 0.31 & $\mathrm{~m}$ \\
\hline$\rho$ & Air density & 1.225 & $\mathrm{~kg} / \mathrm{m}^{3}$ \\
\hline$\eta_{m}$ & Electric motor's efficiency & 0.88 & - \\
\hline$\eta_{\text {gear }}$ & Gearbox efficiency & 0.97 & - \\
\hline
\end{tabular}

\section{2-5 Real-time simulator}

The real-time communication between the rig (electric machines and battery) and the simulation model (vehicle model) is provided using dSPACE system. It consists of a DS1006 processor with the dSPACE 2013b real-time operating system, and DS2202 input-output card interfaced to the user through ControlDesk 5.1 proprietary software platform. All the sensors/actuators are connected to dSPACE through proper wiring.

The simulation files, developed in MATLAB/Simulink environment, are converted to readable codes for the real-time use in the dSPACE simulator using ControlDesk. This is performed using an icon in MATLAB/Simulink (shown in Figure 4), called Build Model, that compiles the files automatically. The variables to be monitored and recorded, are then determined in ControlDesk software. Consequently, the simulation model and all inputs/outputs are transferred and stored in the dSPACE system before the test through the connection between the PC and dSPACE. 


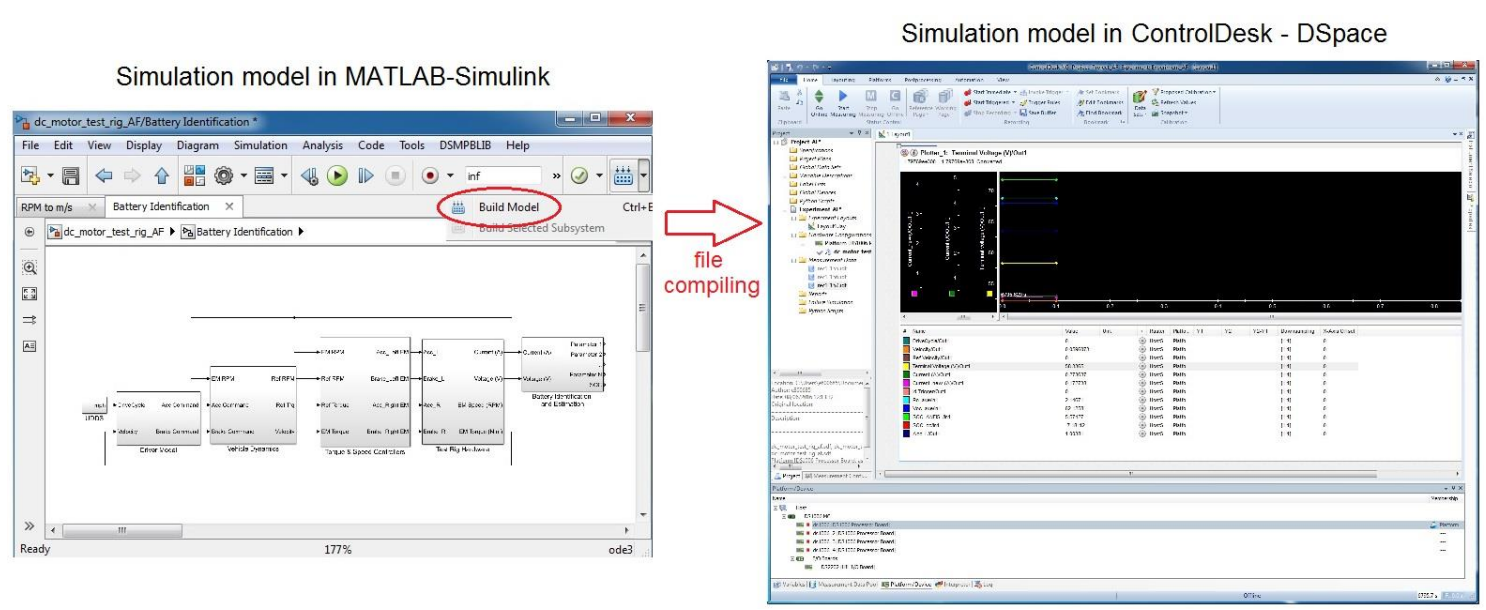

Figure 4: simulation model in MATLAB-Simulink and real-time simulation interface (ControlDesk)

\section{Real-Time Battery Model Identification and SOC Estimation}

A generic framework has been used for real-time battery measurement, model identification and state estimation as demonstrated in Figure 5. Although the idea of this framework was discussed in another study (Fotouhi et al., 2017-a), it has not been tested in real-time before. In this study, embeddable versions of the identification and estimation algorithms were developed and tested in real-time using the HIL test rig. The advantage of this framework is its flexibility to handle different battery types and various targets such as SOC estimation, state-of-health ( $\mathrm{SOH})$ estimation, etc. For more details of the framework presented in Figure 5, please refer to Fotouhi et al., 2017-a. In the first unit of this framework, battery current and voltage are measured as well as the temperature. The measurements are then used by the identification unit to extract battery parameters in real-time. This is called real-time battery modelling. As shown in Figure 5, the number of outputs from this unit is not pre-determined and it depends on the complexity of the model. It is recommended by Fotouhi et al. (2016-a) that the number of parameters is chosen based on what is required for effective state estimation and can change with regard to the battery chemistry. For example, here the target is to estimate SOC of the NiMH battery pack and for this purpose, we just need to have battery pack's open-circuit-voltage (OCV) value. This might not be true for other battery chemistries such as lithium-sulfur cell which has a flat shaped OCV curve as discussed by Fotouhi et al., 2017-a.

The outputs of the identification unit (estimates of unknown parameters) are then used by the estimation unit which uses an artificial intelligent technique (described later in the paper) and is 
trained to find the relationship between the battery parameters and SOC. The effect of the temperature is also taken into account in this unit however; the results of this study are presented for a constant temperature.

It is also worth mentioning how the system works in real-time through the following steps:

1) The measurements are performed continuously at a fixed sampling rate (i.e. 0.1 second in this study).

2) A limited number of latest measured data points are temporarily stored on the BMS board to be used for identification. The data is updated continuously at each time step by eliminating the oldest point and adding the most recent point. For the test explained later in section 4, a two-minute time window was considered. Consequently, the time window contains 1200 data points for each variable (i.e. current and terminal voltage).

3) Identification process is done at certain points (not continuously) using the updated batch of data (1200 data points for each current and voltage). Identification is repeated based on a SOC window. For example, every $0.1 \%$ change in SOC that simply means 1000 repetition of the identification process if we start a test from 100\% SOC and continue until the depleted state $(0 \%$ SOC). Figure 6 shows a battery identification trigger that works based on SOC change in real-time. In this mechanism, an integrator calculates the summation of the energy flow from/into the battery and it resets to zero value every time that the summation value equals or becomes bigger than a threshold (i.e. $0.1 \%$ SOC). The battery model parameters are then updated every time that an identification process is completed; otherwise they keep their previous values.

4) The estimator also works based on a similar (but separate) trigger that updates the estimation results in real-time. Simply speaking, it works like a nonlinear mapping function that determines SOC value by using the identification results (i.e. battery model's parameters). In this study, the estimation trigger acts every $2 \%$ change in SOC that simply means 50 repetition of the estimation process if we start a test from $100 \%$ SOC and continue until the depleted state ( $0 \%$ SOC). The reason of choosing a wider window for estimation is the uncertainties and consequently the fluctuations in the identification results which are filtered by an averaging technique. In this technique, 
SOC estimation interval is determined based on the identification interval and the number of points we need for averaging:

$$
\text { SOC_estimation_interval }=\text { number_of_points } \times \text { identification_interval }
$$

In this study, the average value is calculated using 20 points (identified parameter values every $0.1 \%$ SOC) as discussed in section 4.

In the following sections, more explanation about each part is presented.

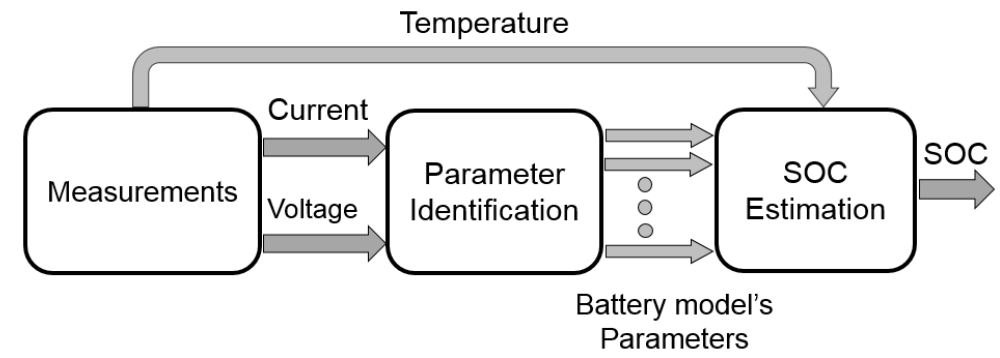

Figure 5: Battery measurement, identification and estimation in real-time

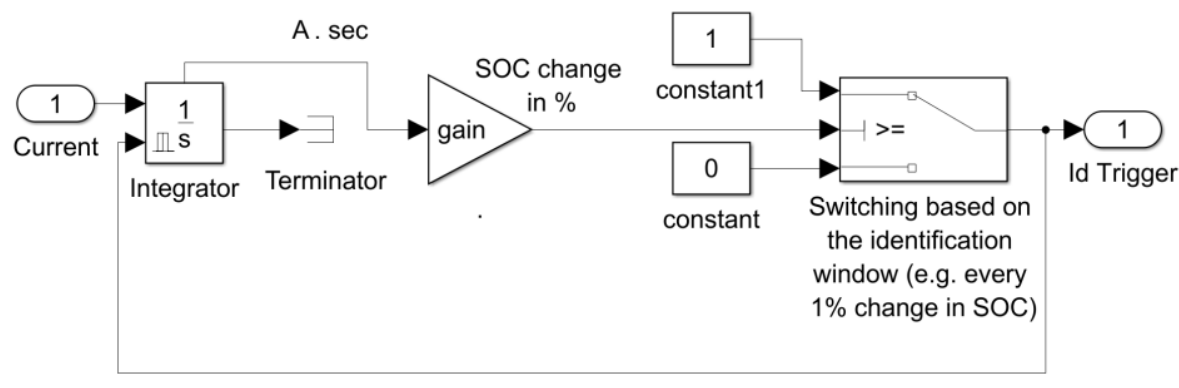

Figure 6: Battery identification trigger, working based on SOC change in real-time

\section{3-1 Battery measurements}

The battery measurement consists of recording load current and terminal voltage during the tests. Temperature is controlled to be fixed at $25{ }^{\circ} \mathrm{C}$ during the experiments by using the thermal chamber (depicted in Figure 1). Test data is stored with a sampling time of 0.1 second. Each test starts from fully charged state and continues until the terminal voltage drops below the cut-off voltage (i.e. $54 \mathrm{~V}$ in this case) which means depleted state. The discharge rate depends on the power demand from the motor controller however, it is limited to $10 \mathrm{~A}$ to protect the battery pack. The setup uses a LEMTM 5V maximum output \pm 19.2 A current transducer (model number LTS 6NP) for current measurement. The voltage output of the current transducer is fed to the analog-to-digital (AD) input of the dSPACE setup. The battery terminal voltage is measured 
using a simple resistive potential divider circuit. The divider resistors are selected such that the current through them is less than $0.1 \mathrm{~mA}$ and the output voltages are less than $10 \mathrm{~V}$, which is a limit of dSPACE system.

\section{3-2 Battery parameter identification}

A system identification technique is used to find the battery parameters based on input-output battery measurements which are current and terminal voltage. Various ECN model structures and fitting algorithms can be used for this purpose depending on the required level of complexity and speed in each application. In the present case that the final target is the NiMH pack's SOC estimation, the results presented by Fotouhi et al. (2017-a) demonstrate that the battery pack's OCV would be sufficient. Consequently, the only unknown parameter (referring to Figure 5) which should be obtained during the identification process is OCV in this case. For this purpose, an internal resistance model ( $R_{\text {int }}$ model) (Johnson, 2002) is used which contains a voltage source and a resistor in series.

After selecting the battery model structure, the unknown parameters should be found during the identification process. The parameter vector $(\theta)$ is determined so that the prediction error $(\varepsilon)$ is minimized, defined as follows (Fotouhi et al., 2016-a):

$$
\varepsilon\left(t_{k}, \theta\right)=y\left(t_{k}\right)-\hat{y}\left(t_{k} \mid t_{k-1} ; \theta\right)
$$

where $y\left(t_{k}\right)$ is the measurement data at time $k$ and $\hat{y}\left(t_{k} \mid t_{k-1} ; \theta\right)$ is the model's prediction at time $k$ using the parameters $\theta$. Using root mean square error (RMSE) criterion, the following fitness function is minimized;

$$
R M S E=\left[\frac{1}{N} \sum_{k=1}^{N}\left|\varepsilon\left(t_{k}, \theta\right)\right|^{2}\right]^{\frac{1}{2}}
$$

In this case, the battery model's parameters are optimized so that the least difference between the measured terminal voltage and the model's output is achieved so we have:

$$
R M S E=\frac{1}{\sqrt{n}}\left(\sum_{i=1}^{n}\left(V_{t, i}-\hat{V}_{t, i}\right)^{2}\right)^{0.5}
$$


where $V_{t}$ and $\hat{V}_{t}$ are measured and estimated values of the battery terminal voltage respectively. Using $R_{\text {int }}$ model, the estimated value can be obtained as follows;

$$
\hat{V}_{t}=V_{O C}-R_{\mathrm{int}} \cdot I
$$

In this case, the RMSE is a function of the two variables, $V_{O C}$ and $R_{\mathrm{int}}$. To minimize the RMSE, optimal values of the variables are obtained by putting the gradients equal to zero that gets the following closed-form formulas (Fotouhi et al., 2016-a):

$$
\begin{gathered}
R_{\mathrm{int}}=\left(\frac{\sum_{i=1}^{n} V_{t, i} I_{i}}{\sum_{i=1}^{n} I_{i}}-\frac{\sum_{i=1}^{n} V_{t, i}}{n}\right) /\left(\frac{\sum_{i=1}^{n} I_{i}}{n}-\frac{\sum_{i=1}^{n} I_{i}^{2}}{\sum_{i=1}^{n} I_{i}}\right) \\
V_{O C}=R_{\mathrm{int}} \cdot \frac{\sum_{i=1}^{n} I_{i}^{2}}{\sum_{i=1}^{n} I_{i}}+\frac{\sum_{i=1}^{n} V_{t, i} I_{i}}{\sum_{i=1}^{n} I_{i}}
\end{gathered}
$$

The above formulas give us optimal values of $V_{O C}$ and $R_{\mathrm{int}}$ corresponding to the least RMSE. Some points that should be considered when using this analytical solution are:

1) The analytical solution is obtained for the $R_{\text {int }}$ model and is not valid for other ECN models.

2) The formula can be used for any battery type (not limiting to NiMH battery).

3) It gives the average values of $V_{O C}$ and $R_{\text {int }}$ by using charge and discharge data at the same time.

\section{3-3 Battery SOC estimation}

An adaptive neuro-fuzzy inference system (ANFIS) was used for battery pack's SOC estimation in this study. This is about the last part of the whole framework presented in Figure 5. Here ANFIS performs as an estimator which uses OCV and temperature as the inputs to predict SOC as the output as shown in Figure 7. Indeed, ANFIS needs to be trained to learn the relationship of the battery parameters (i.e. OCV in this case) and temperature with SOC. The battery parameters are identified and used in real-time as an indicator of SOC. So, a nonlinear 
mapping tool is required, that is ANFIS here. (It should be noted that other mapping tools may also be applicable as well as ANFIS.)

It is demonstrated in the literature that battery's performance is a function of different factors such as SOC, state of health $(\mathrm{SOH})$, temperature, etc. So, generally cell's parameters $\left(P_{i}\right)$ are functions of those variables as follows:

$$
P_{i}=f_{i}(S O C, S O H, T, \ldots), \quad i=1 . . n
$$

The number of parameters and variables which are used in a battery model depends on the required precision. In some cases, high-fidelity models are needed whereas in other cases, a fast low-fidelity model is desired. In this study, a simple and fast model is needed for real-time SOC estimation. The idea of SOC estimation is to find an inverse function (nonlinear mapping) which is able to predict SOC by using the identified parameters. Being aware of the influence of $\mathrm{SOH}$ on our results, we assume fixed value for this variable and plan to extend our algorithms in our future works. So, the following equation is used for the sake of simplicity:

$$
P_{i}=f_{i}(S O C, T), \quad i=1 . . n
$$

The battery parameters $\left(P_{i}\right)$ are obtained by using the system identification algorithm and then a nonlinear function like $\mathrm{g}$ in bellow (i.e. ANFIS) is utilized for SOC estimation.

$$
S O C=g\left(P_{1}, P_{2}, . ., P_{n}\right)
$$

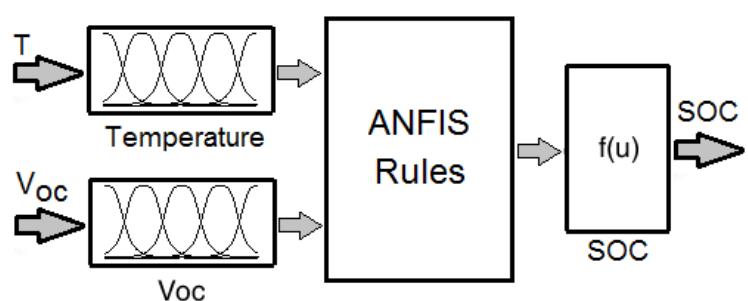

Figure 7: ANFIS structure used for NiMH pack's SOC estimation

ANFIS is a powerful tool for modelling, prediction and control by integrating synergy of the artificial neural network and the fuzzy logic. A fuzzy set (which works based on fuzzy logic) is an efficient tool for modelling of a phenomenon related to human knowledge by using linguistic labels that are embedded in a number of membership functions. A fuzzy inference system (FIS) is a collection of fuzzy if-then rules which contains human expertise and knowledge. Because a FIS doesn't have learning and adaptation capabilities, neuro-fuzzy techniques have been developed in order to add this functionality to a FIS as well. A hybrid learning approach is used in ANFIS 
structure that combines the gradient-descent and the least squares methods for the parameter tuning (Jang et al 1997). ANFIS has been used in a wide range of applications and particularly, it has been used for battery SOC estimation in previous studies (Cai et al 2003; Tsai et al 2012). For more details on the theory of ANFIS and the training algorithms, the reader is suggested to refer to Jang et al (1997).

The advantages of the proposed framework (presented in Figure 5) can be summarized as follows:

(i) It is a flexible framework that can be applied to various battery types. It is demonstrated by Fotouhi et al. (2017-b) that the framework can even handle the flat OCV curve of a lithiumsulfur cell. Because of the observability issues, most of the existing estimation techniques are not applicable for lithium-sulfur cell (Fotouhi et al. 2017-b).

(ii) Unlike coulomb-counting, it can start from any initial SOC value and no initial condition data is needed to run the estimator.

(iii) Unlike coulomb-counting, the whole battery capacity is not needed for SOC calculation.

(iv) Unlike recursive adaptive filters, no time is needed for convergence of the algorithm at the beginning.

(v) The proposed method is simple and fast enough to be used in real-time applications. In addition, ANFIS models are adaptive and can be retuned very fast.

For validation of the estimations, 'ideal' coulomb-counting (CC) technique is used as a benchmark. CC is a theoretical method which cannot be used in practice because of its restrictions however; it is a good benchmark for checking other techniques (Kutluay et al., 2005). In $\mathrm{CC}$, battery SOC is obtained by integrating the current over time. Assuming $S O C_{0}$ as the initial SOC at time $t_{0}$, the battery pack's SOC at time $t$ is:

$$
S O C=S O C_{0}-\left(\int_{t_{0}}^{t} \frac{\eta i(\tau)}{C_{t}} d \tau\right), \quad 0<S O C<1
$$

where $i(t)$ is the battery current (A) (assumed positive for discharging and negative for charging). $\eta$ is the battery's coulombic efficiency (dimensionless) and $C_{t}$ is the total capacity (As). In this representation, the SOC value is a number between 0 and 1 that 0 indicates a fully depleted state and 1 represents a fully charged state. 


\section{Results Analysis}

In a case study, a $64.8 \mathrm{~V}$ NiMH battery pack was tested using the HIL test rig and the proposed algorithms were used in real-time. The test was performed according to UDDS standard (DieselNet, 2017), under constant temperature at $25^{\circ} \mathrm{C}$, starting from fully-charged state $(100 \%$ $\mathrm{SOC}$ ) to fully-discharged state (zero SOC) when the terminal voltage drops below $54 \mathrm{~V}$ (i.e. the cut-off voltage of the battery pack). Figure 8 demonstrates how the battery terminal voltage has changed over 5 consecutive UDDS cycles. Since the sampling rate was $10 \mathrm{~Hz}$, the time in the figures is presented in decisecond ( 0.1 second).

The driver model followed the driving cycle by generating an acceleration command when the actual velocity is less than the reference velocity. Rotational speed of the electric motors was measured and a velocity tracking error was calculated. The reference velocity and measured velocity are illustrated in Figure 8 (for the whole test) and Figure 9 (for the first cycle only), demonstrating a good tracking performance. Variations of battery pack's current and voltage are also depicted in Figure 8 (for the whole test) and in Figure 9 and Figure 10 (for the first cycle only). As shown in Figure 8 to Figure 10, the measurements are quite noisy due to using inexpensive sensors. This is done by purpose because we were interested in providing a real challenging platform for BMS algorithms testing. Such a testing platform is similar to a real automotive application with budget constraints. In addition, we believe that testing and validation of the proposed BMS algorithms using such noisy data would be sufficient to be sure about other 'less challenging' situations.

In the test presented in Figure 8, speed following of the traction motor is performed without braking (i.e. no negative command, no energy regeneration). In this scenario, an acceleration command was generated when increase in speed is required and zero acceleration when decrease in speed is required. This works in practice because of the friction in the whole system and also small rotational momentum of the system (vehicle inertia is neglected for the sake of simplicity).

Although the effect of regenerative braking is not considered in this study, the algorithms are able to deal with instantaneous charging pulses (between the discharge pulses) as well. However, a continuous charging profile needs particular considerations. 

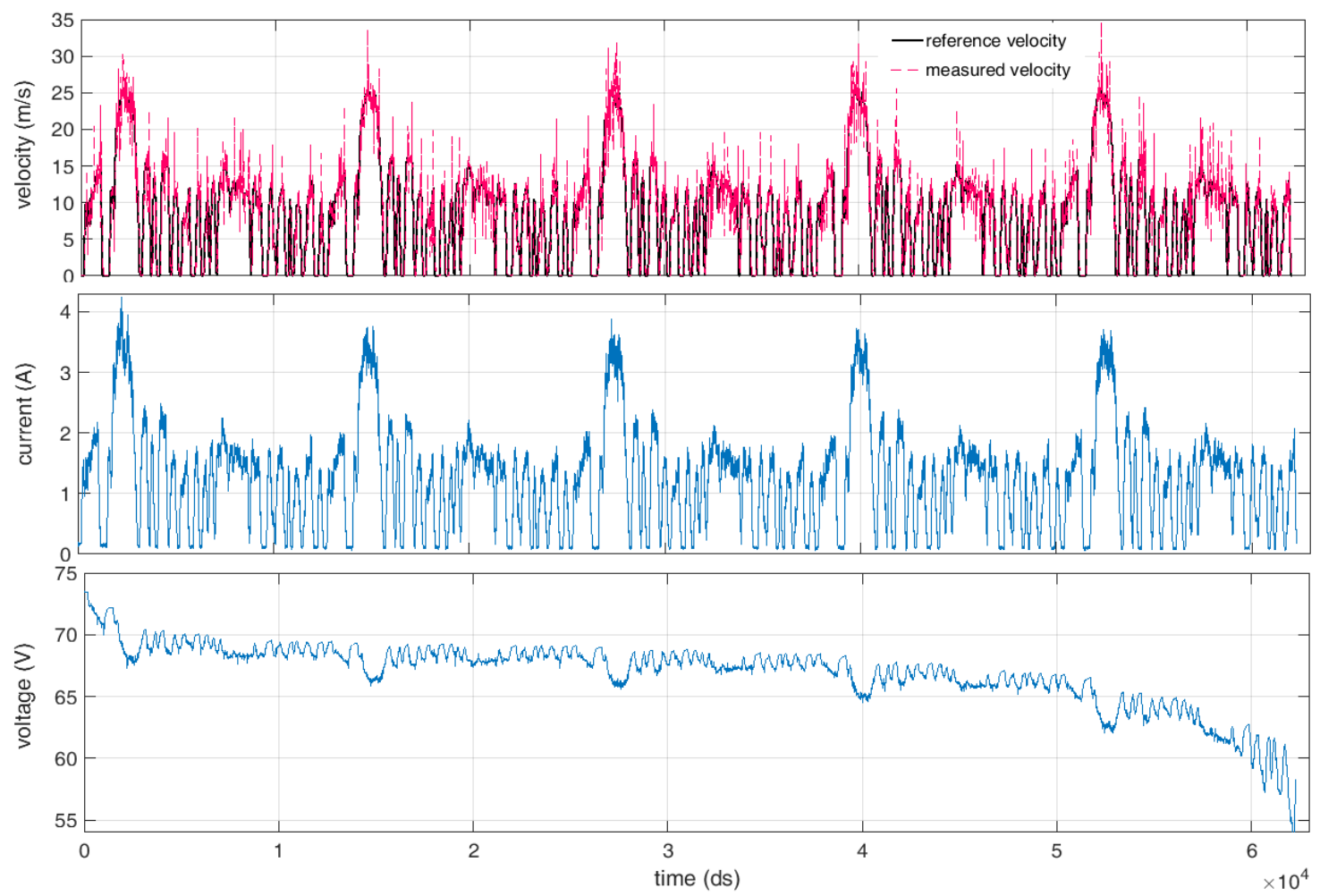

Figure 8: velocity and battery measurements for the whole test
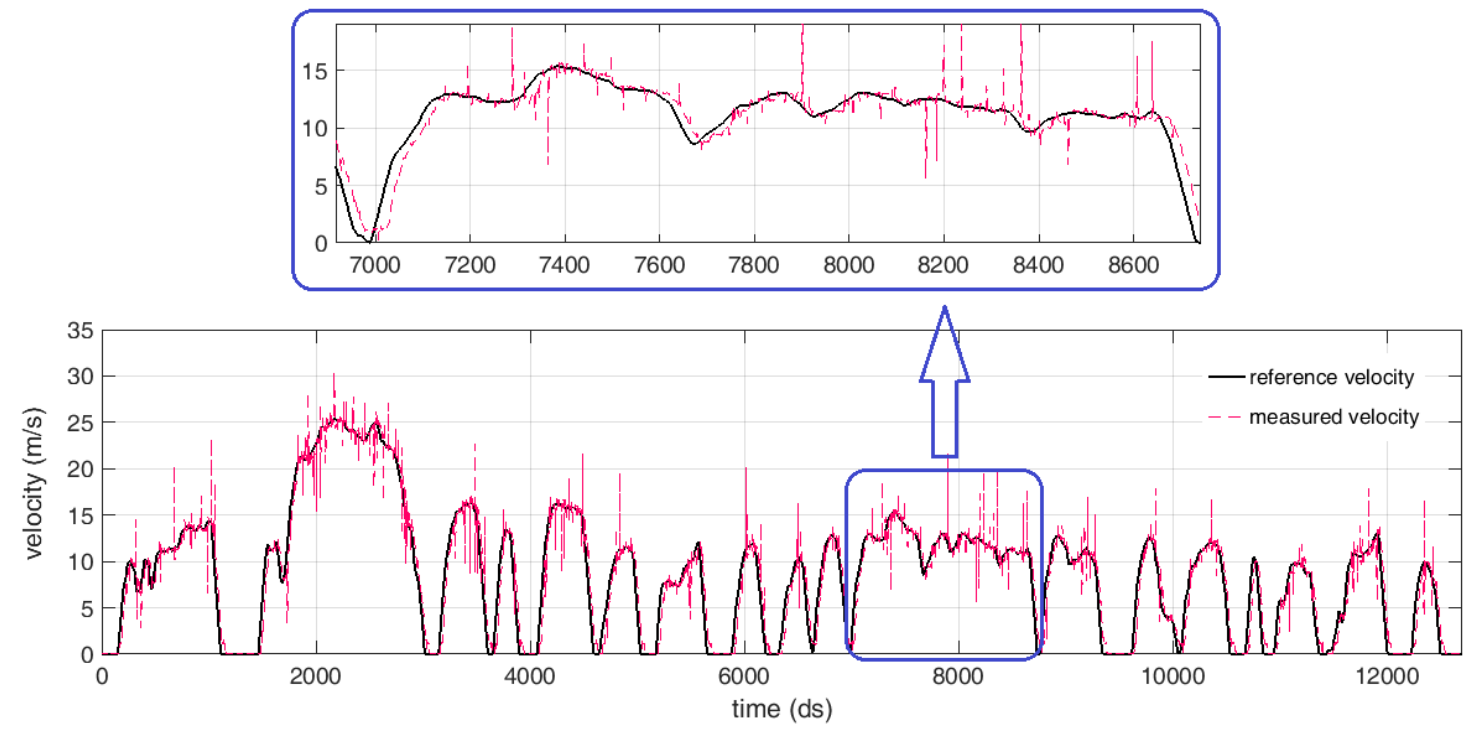

Figure 9: reference and measured velocity profiles for the first cycle 

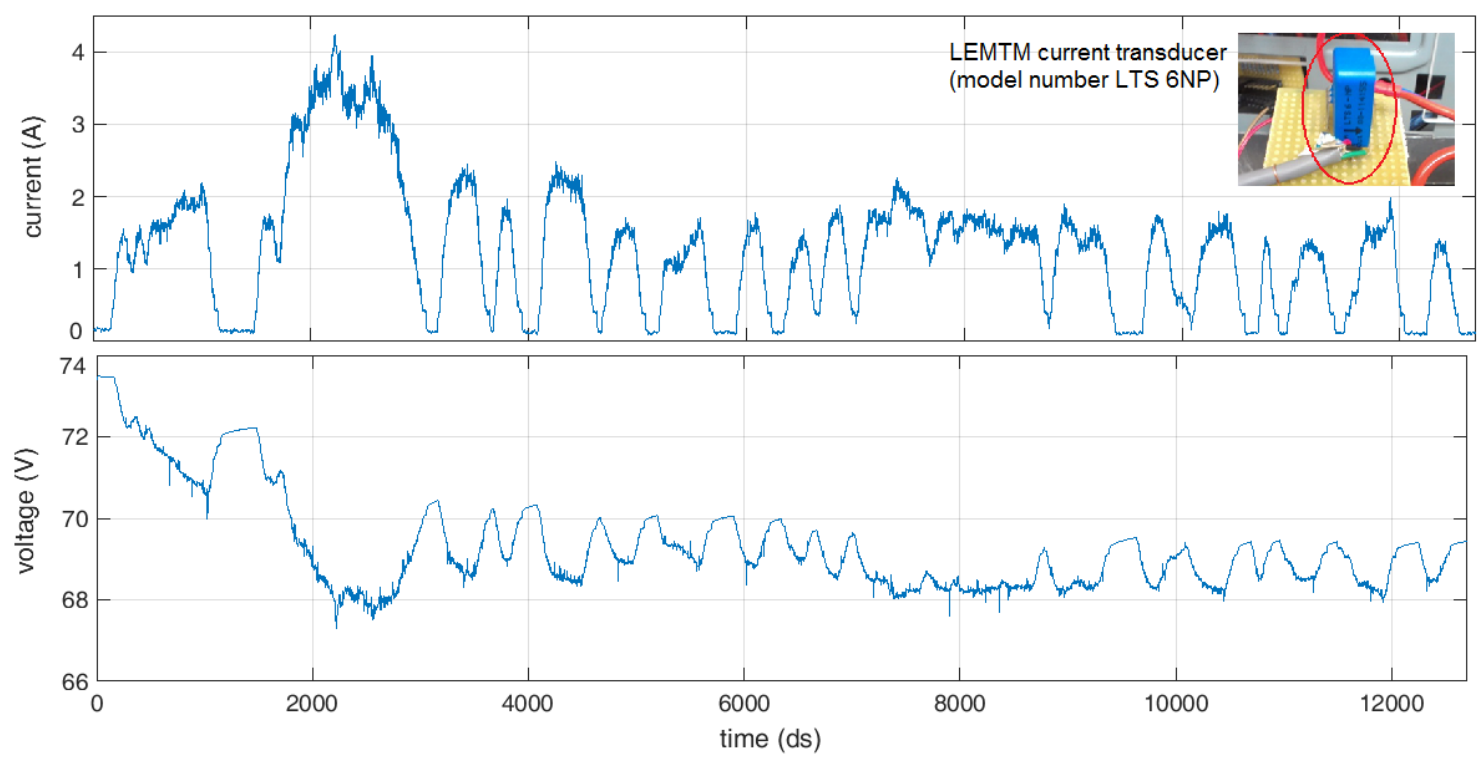

Figure 10: battery current and terminal voltage profiles for the first cycle

Identification and estimation results are presented in Figure 11 including battery internal resistance and OCV as discussed in section 3.2 and battery SOC estimation using ANFIS explained in section 3.3. According to the results demonstrated in Figure 11, the identification and estimation algorithms perform well against the high level of noise (caused by using low-cost sensors) which is designed by purpose to test the algorithms in the worst scenario.

Identification was repeated every $0.1 \%$ change in SOC using a two-minute time window. One of the main advantages of the proposed identification formula is its high speed that makes it suitable for real-time applications. In this case, the identification process was repeated every 6 seconds however, it is possible to run it much faster (Fotouhi et al., 2016-a). On the other hand, the identification algorithm has limitations as well: (i) as shown in Figure 11, there are fluctuations in the identification results depending on the current profile. There is an error in the identification results especially at high discharge rate. (ii) the algorithm has also limitation during continuous discharge since identifying battery resistance and OCV is challenging in this case. No drop or jump is detectable during a continuous discharge pulse. To overcome these limitations, an averaging technique is used by repeating the identification faster and doing the estimation in a longer window. SOC estimation was performed using the last 20 identification points which means every $2 \%$ change in SOC in this case. The reason of using a number of identification points ( 20 points in this case) for every estimation attempt is to make the estimation results more 
robust against the identification uncertainties. Consequently, one or two faulty points in the identification process can not affect the estimation result very much.

Regarding the estimation results shown in Figure 11, the theoretical (ideal) coulomb-counting was used as a benchmark for evaluation of the ANFIS method. It is worth mentioning that the 'ideal' coulomb-counting is not applicable in a real application because of its practical limitations such as cumulative noise effect (due to the integration of the current signal as presented in equation 14) and the need for 'known' initial condition (Kutluay et al., 2005). In Figure 11, coulomb-counting is calculated based on an assumption that we know the initial SOC value. However, the ANFIS method is able to recognize the initial state by using the OCV value. On the other hand, each estimation try by ANFIS is independent of its previous try. So, there is no cumulative error when using ANFIS technique. The drawback of the ANFIS technique is its robustness against the identification accuracy (OCV values in this case) that can cause to fluctuations in the estimation results.
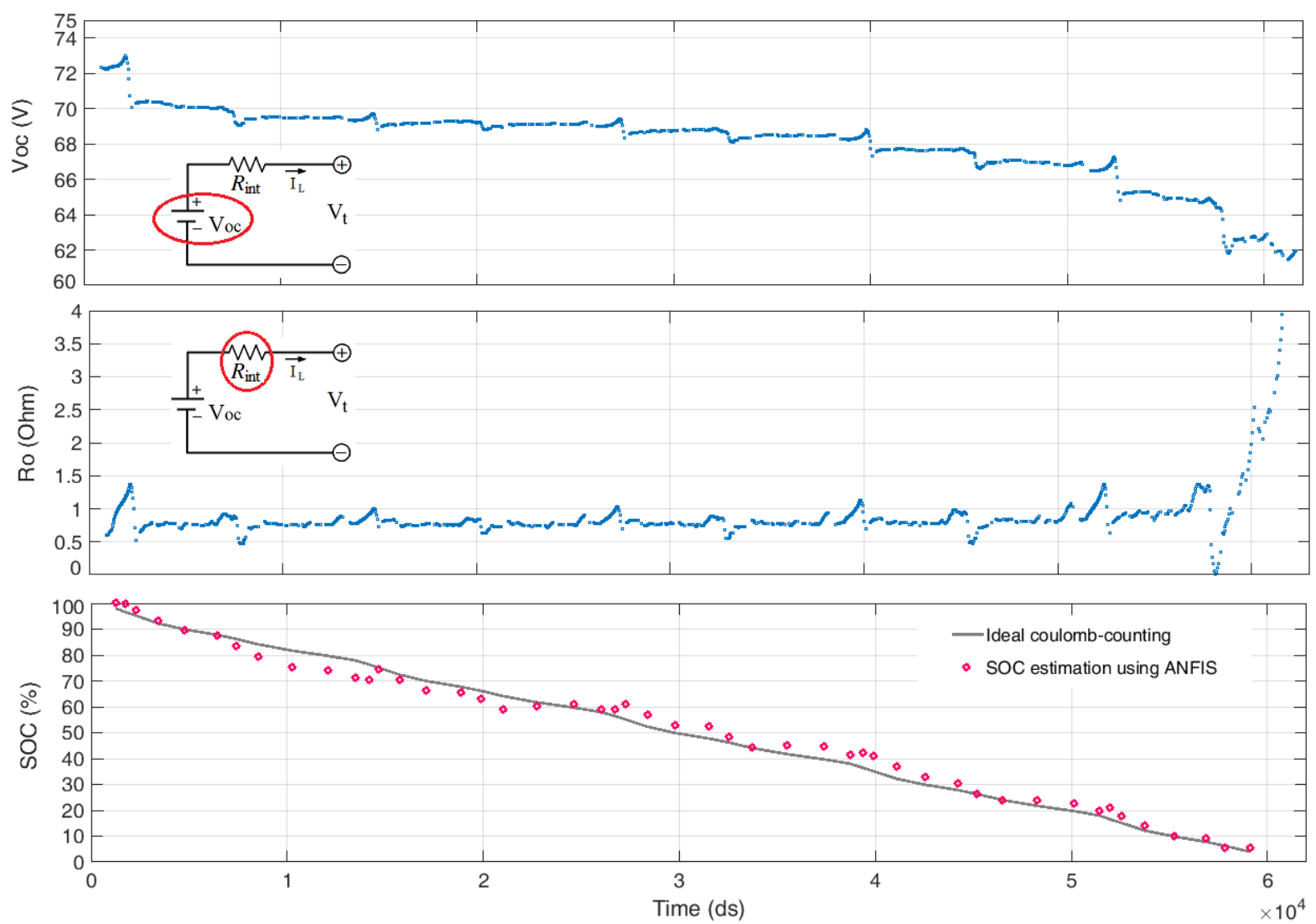

Figure 11: real-time battery parameter identification and SOC estimation during UDDS test 


\section{Conclusions}

Performance of the proposed BMS algorithm was tested properly using the HIL test rig, representing a scaled-down electric vehicle powertrain system. Using such a HIL test rig for BMS algorithm development, is a quite useful approach to evaluate the proposed algorithms in a more realistic scenario by considering the effects of noise and uncertainties. A generic framework was explained and tested for real-time battery model identification and state estimation. Battery internal resistance and open-circuit voltage were obtained in real-time using current and voltage measurements. The main advantage of the proposed identification algorithm is its simplicity and speed that makes it suitable to be easily embedded on battery management boards. Another advantage of the proposed framework is its flexibility that makes it usable for other applications like state-of-health estimation and other battery types too. Drawbacks of the proposed BMS algorithm were also investigated. There were fluctuations in the identification results especially at high discharge rate. The identification algorithm has also limitation during continuous discharge pulses without any drop or jump in between. Using the solutions like averaging technique and ANFIS, battery SOC estimation result was satisfactory against the limitations of the identification unit.

\section{References}

Antaloae C., Marco J., Assadian F. (2012) 'A Novel Method for the Parameterization of a Li-Ion Cell Model for EV/HEV Control Applications', IEEE Transactions on Vehicular Technology, 61(9), pp. 3881-3892.

Barreras J. V., Fleischer C., Christensen A. E., Swierczynski M., Schaltz E., Andreasen S. J., and Sauer D. U. (2016) 'An Advanced HIL Simulation Battery Model for Battery Management System Testing', IEEE transactions on industrial applications, 52 (6), 5086-5099.

Bose B. K. (2001) 'Modern Power Electronics and AC Drives', Prentice Hall Publishers.

Cai C.H., Du D., Liu Z.Y., (2003) 'Battery State-of-Charge (SOC) Estimation Using Adaptive Neuro-Fuzzy Inference System (ANFIS),' The IEEE international Conference on Fuzzy Systems, vol.2, pp. 1068-1073.

Chiasson J., Vairamohan B. (2005) 'Estimating the state of charge of a battery', IEEE Transactions on Control Systems Technology, 13(3), pp. 465-470. 
Choi G., Jahns T. M. (2013) 'Design of Electric Machines for Electric Vehicles Based on Driving Schedules', Proc. IEEE Electric Machines \& Drives, pp.54-61.

Cuma M. U., Koroglu T., Renew, Sust. Energ. Rev. 42 (2015) 517.

DieselNet (2017) [online] http://www.dieselnet.com/standards/cycles/ftp72.html

Edwards C., Spurgeon S., Sliding Mode Control: Theory and Applications, London, Taylor and Francis, 1998.

Fetcenko M. A., Ovshinsky S. R., Reichman B., Young K., Fierro C., Koch J., Zallen A., Mays W., Ouchi T. (2007) 'Recent advances in NiMH battery technology', Journal of Power Sources, 165, pp. 544-551.

Fotouhi A, Auger D J, Propp K, Longo S, (2014) Simulation for prediction of vehicle efficiency, performance, range and lifetime: A review of current techniques and their applicability to current and future testing standards, 5th IET Hybrid and Electric Vehicles Conference (HEVC), London, UK, November 5-6.

Fotouhi A., Auger D. J., Propp K., Longo S. (2016-a) 'Accuracy versus Simplicity in Online Battery Model Identification', IEEE Transactions on Man, Systems and Cybernetics.

Fotouhi A, Auger D. J., Propp K., Longo S., Wild M. (2016-b) 'A review on electric vehicle battery modelling: From Lithium-ion toward Lithium-Sulphur', Renewable and Sustainable Energy Reviews, 56, pp. 1008-1021.

Fotouhi A., Auger D. J., Propp K., Longo S. (2017-a), Electric Vehicle Battery Parameter Identification and SOC Observability Analysis: NiMH and Li-S Case Studies, IET Power Electronics, 10(11), pp. 1289-1297.

Fotouhi A., Auger D. J., Propp K., Longo S., (2017-b), Lithium-Sulfur Battery State-of-Charge Observability Analysis and Estimation, IEEE Transactions of Power Electronics. DOI: 10.1109/TPEL.2017.2740223.

Gao D. W., Mi C., Emadi A. (2007) 'Modeling and Simulation of Electric and Hybrid Vehicles', in Proceedings of the IEEE, vol. 95, no. 4, pp. 729-745.

Hayes J. G., de Oliveira R.P.R., Vaughan S., Egan M.G. (2011) 'Simplified Electric Vehicle Power Train Models and Range Estimation', Proc. IEEE Vehicle Power and Propulsion, pp.15 .

He H., Xiong R., Fan J. (2011) 'Evaluation of lithium-ion battery equivalent circuit models for state of charge estimation by an experimental approach', Energies, 4, pp. 582-598. 
He W., Williard N., Chen C., Pecht M., Microelectron. Reliab. 53(6) (2013) 840.

Jang J. S. R., Sun C. T., Mizutani E., (1997) 'Neuro-fuzzy and soft computing: a computational approach to learning and machine intelligence' Prentice-Hall, Inc. Upper Saddle River, NJ, USA.

Johnson V. H. (2002) 'Battery performance models in ADVISOR', J. Power Sources, 110, pp. 321-329.

Kelly K. J., Mihalie M., Zolot M. (2002) 'Battery usage and thermal performance of the Toyota Prius and Honda Insight during chassis dynamometer testing', IEEE Battery Conference on Applications and Advances, Long Beach, CA, USA.

Kim I. S. (2010), IEEE Transaction on Power Electronics, 25, p. 1013.

Kutluay, Cadirci Y., Ozkazanc Y. S., Cadirci I. (2005) 'A new online state-of-charge estimation and monitoring system for sealed lead-acid batteries in Telecommunication power supplies', IEEE Transactions on Industrial Electronics, 52, pp. 1315-1327.

Lu L., Han X., Li J., Hua J., Ouyang M., (2013), Journal of Power Sources 226, p. 272.

Lukic S. M., Emado A. (2003) 'Modeling of electric machines for automotive applications using efficiency maps', Electrical Insulation Conference and Electrical Manufacturing and Coil Winding Technology Conference, pp. 543-550.

Marc T., Oliver B., Dirk B. U.S. (2008) 'Development of a voltage-behavior model for NiMH batteries using an impedance-based modeling concept', J. Power Sources, 175, pp. 635-643.

Piller S., Perrin M., Jossen A., (2001), Journal of Power Sources, 96, p. 113.

Plett G. L., (2004), Journal of Power Sources 134, p. 262.

Schweighofer B. R. K., Brasseur G. (2003) 'Modeling of high power automotive batteries by the use of an automated test system', IEEE Transactions on Instrumentation and Measurement, 52 (4), pp. 1087-1091.

Thrun S., Burgard W., Fox D., Probabilistic Robotics. MIT Press, 2005.

Tsai M.F., Peng Y.Y., Tseng C.S., Li N.S., (2012) 'Modeling and Estimation of State of Charge for Lithium-Ion Batteries Using ANFIS Architecture', IEEE International Symposium on Industrial Electronics (ISIE), Hangzhou, pp. 863-868.

Wang L. (2014) 'Development of a Hardware-In-the-Loop Simulator for Battery Management Systems', MSc Thesis, The Ohio State University. 
Welch G., Bishop G., An Introduction to the Kalman Filter, Tech. Report, University of North Carolina, Chapel Hill, NC, USA, 1995.

Ziegler J.G, Nichols N. B. (1942) 'Optimum settings for automatic controllers', Transactions of the ASME, Vol. 64, pp. 759-768.

\section{Acknowledgements}

This research was undertaken as part of two projects: the 'Revolutionary Electric Vehicle Battery' (REVB) project, co-funded by Innovate UK /EPSRC (TS/L000903/1 and EP/ L505286/1), and the 'Understanding Future Vehicles' project funded by EPSRC (EP/I038586/1). Data underlying this study can be accessed through the Cranfield University repository at http://dx.doi.org/10.17862/cranfield.rd.5687983. 


\section{A hardware-in-the-loop test rig for development of electric vehicle battery identification and state estimation algorithms}

Fotouhi, Abbas

Inderscience

Fotouhi A, Propp K, Samaranayake L, et al., (2018) A hardware-in-the-loop test rig for development of electric vehicle battery identification and state estimation algorithms.

International Journal of Powertrains, Volume 7, Issue 1-3, 2018, pp. 227-248

http://dx.doi.org/10.1504/IJPT.2018.090391

Downloaded from Cranfield Library Services E-Repository 